VOL. 75 (2007) [321-329]

\title{
UNIQUE EXTREMALITY, LOCAL EXTREMALITY AND EXTREMAL NON-DECREASABLE DILATATIONS
}

\author{
GuOWU YaO
}

\begin{abstract}
Given a quasi-symmetric self-homeomorphism $h$ of the unit circle $S^{1}$, let $Q(h)$ be the set of all quasiconformal mappings with the boundary correspondence $h$. In this paper, it is shown that there exists certain quasi-symmetric homeomorphism $h$, such that $Q(h)$ satisfies either of the conditions,
\end{abstract}

(1) $Q(h)$ admits a quasiconformal mapping that is both uniquely locallyextremal and uniquely extremal-non-decreasable instead of being uniquely extremal;

(2) $Q(h)$ contains infinitely many quasiconformal mappings each of which has an extremal non-decreasable dilatation.

An infinitesimal version of this result is also obtained.

\section{INTRODUCTION}

Let $\Delta$ be the unit disk $\{z:|z|<1\}$ in the complex plane $\mathbb{C}$. Given a quasisymmetric homeomorphism $h$ of the unit disk $S^{1}$ onto itself, we denote by $Q(h)$ the class of all quasiconformal mappings from $\Delta$ onto itself with the boundary correspondence $h$. A quasiconformal mapping $f_{0} \in Q(h)$ is said to be an extremal mapping for the boundary correspondence $h$ if it minimises the maximal dilatations of $Q(h)$, that is,

$$
K\left[f_{0}\right]=K[h]:=\inf \{K[f]: f \in Q(h)\}
$$

where $K[f]$ is the maximal dilatation of $f . f$ is uniquely extremal if it is extremal and if there are no other extremal mappings for its boundary values; the alternative is that $f$ is non-uniquely extremal.

The notion of non-decreasable was first introduced by Reich in [7] to investigate the unique extremality of quasiconformal mappings between the unit disks with given

\section{Received 18th October, 2006}

The author would like to thank Professor Edgar Reich for sending him his inspiring paper [7]. The research was supported by the National Natural Science Foundation of China (Grant No. 10401036) and a Foundation for the Author of National Excellent Doctoral Dissertation (Grant No. 200518) of PR China.

Copyright Clearance Centre, Inc. Serial-fee code: 0004-9727/07 \$A2.00+0.00. 
boundary values. An element $f$ in $Q(h)$ has a non-descreasable dilatation (or $f$ is called non-decreasable), if the hypothesis that $g$ is also in $Q(h)$ together with the condition,

$$
|\nu(z)| \leqslant|\mu(z)| \text { almost everywhere in } \Delta \text {, }
$$

imply that $f=g$, where $\mu$ and $\nu$ are the Beltrami coefficients of $f$ and $g$, respectively. Obviously, if $f$ is uniquely extremal, then it has non-decreasable dilatation. But the converse is not true. So the conception of quasiconformal mappings with non-decreasable dilatations is a generalisation of uniquely extremal quasiconformal mappings.

In [8], Shen and Chen proved that, if $Q(h)$ does not contain a conformal mapping, then it must contain infinitely many elements with non-decreasable dilatations. So it is more interesting to investigate extremal quasiconformal mappings with non-decreasable dilatations; accordingly, such non-decreasable dilatations are called extremal ones. It is still an open problem whether an extremal quasiconformal mapping with non-decreasable dilatation always exists in $Q(h)$.

Following [9], a quasiconformal mapping $f$ of $\Delta$ is said to be locally extremal if for any domain $G \subset \Delta$ the mapping $f$ is extremal in $G$ with respect to its boundary values. The complex dilatation $\mu$ of $f$ is then called locally extremal dilatation. Generally speaking, both the uniqueness and the existence of locally extremal quasiconformal mappings in $Q(h)$ are not clear. An example due to Reich ([5], or see [11]) shows that local extremality does not imply unique extremality.

Obviously, if $f$ is uniquely extremal, then $f$ is the quasiconformal mapping in $Q(h)$ that is both uniquely locally-extremal and uniquely extremal-non-decreasable. Conversely, one might ask

Problem 1. If $f$ in $Q(h)$ is the quasiconformal mapping that is both uniquely locallyextremal and uniquely extremal-non-decreasable, is it then uniquely extremal?

REMARK 1. If $f$ has an extremal-non-decreasable dilatation $\mu(z)$ with the property that $|\mu(z)|=$ constant almost everhwyere in $\Delta$, then it is obviously uniquely extremal; the converse is not true, as is a well-known result in [2]. There are a lot of examples (see [8, Corollary 3.1]) to show that uniqueness of extremal non-decreasable dilatations does not imply unique extremality.

On the other hand, it is natural to pose the following problem.

Problem 2. Does there exist $h$ such that $Q(h)$ contains infinitely many extremal quasiconformal mappings with non-decreasable dilatations?

Our main result Theorem 1 gives a negative answer to Problem 1 and a positive one to Problem 2, respectively. Meanwhile, an infinitesimal version is obtained for the tangent space of the universal Teichmüller space. 


\section{Preliminaries}

Let $\mathfrak{D}$ be a domain in the complex plane $\mathbb{C}$ with at least two boundary points and let $M(\mathfrak{D})$ be the open unit ball of $L^{\infty}(\mathfrak{D})$. Every element $\mu \in M(\mathfrak{D})$ can be regarded as an element in $L^{\infty}(\mathbb{C})$ by putting $\mu$ equal to zero in the outside of $\mathfrak{D}$. Every $\mu \in M(\mathfrak{D})$ induces a global quasiconformal self-mapping $f$ of the plane which solves the Beltrami equation [1],

$$
f_{\bar{z}}(z)=\mu(z) f_{z}(z),
$$

and $f$ is defined uniquely up to postcomposition by a complex affine map of the plane. Conversely, any quasiconformal mapping $f$ defined on $\mathfrak{D}$ has a Beltrami coefficient $\mu(z)=f_{\bar{z}}(z) / f_{z}(z)$ in $M(\mathfrak{D})$.

Two Beltrami coefficients $\mu, \nu \in M(\mathfrak{D})$ are equivalent if they induce quasiconformal mappings $f$ and $g$ by (2.1) such that there is a conformal map $c$ from $f(\mathfrak{D})$ to $g(\mathfrak{D})$ and an isotopy through quasiconformal mappings $h_{t}, 0 \leqslant t \leqslant 1$, from $\mathfrak{D}$ to $\mathfrak{D}$ which extend continuously to the boundary of $\mathfrak{D}$ such that

1. $h_{0}(z)$ is identically equal to $z$ on $\mathfrak{D}$,

2. $h_{1}$ is identically to $g^{-1} \circ c \circ f$, and

3. $h_{t}(p)=g^{-1} \circ c \circ f(p)$ for any $p \in \partial \mathfrak{D}$.

The equivalence relation partitions $M(\mathfrak{D})$ into equivalence classes and the space of equivalence classes is by definition the Teichmüller space $T(\mathfrak{D})$ of $\mathfrak{D}$.

Given $\mu \in M(\mathfrak{D})$, we denote by $[\mu]$ the set of all elements $\nu \in M(\mathfrak{D})$ equivalent to $\mu$, and set

$$
k_{0}([\mu])=\inf \left\{\|\nu\|_{\infty}: \nu \in[\mu]\right\} .
$$

We say that $\mu$ is extremal (in $[\mu])$ if $\|\mu\|_{\infty}=k_{0}([\mu]), \mu$ is uniquely extremal if $\|\nu\|_{\infty}$ $>k_{0}([\mu])$ for any other $\nu \in[\mu]$; the alternative is that $\mu$ is non-uniquely extremal. We say that $\mu$ is non-decreasable if for any other $\nu \in[\mu]$, the set on which $|\nu(z)|>|\mu(z)|$ has positive measure. Obviously, $\mu$ is non-decreasable if it is uniquely extremal.

For any $\mu$, define $h^{*}(\mu)$ to be the infimum over all compact subsets $F$ contained in $\mathfrak{D}$ of the essential supremum norm of the Beltrami coefficient $\mu(z)$ as $z$ varies over $\mathfrak{D} \backslash F$. Define $h([\mu])$ to be the infimum of $h^{*}(\mu)$ taken over all representatives $\mu$ of the class $[\mu]$. It is obvious that $h([\mu]) \leqslant k_{0}([\mu])$. Following [3], we call a point $[\mu]$ in $T(\mathfrak{D})$ a Strebel point if $h([\mu])<k_{0}([\mu])$.

Let $A(\mathfrak{D})$ be the space of integrable holomorphic quadratic differentials $\varphi$ on $\mathfrak{D}$ and let $A_{1}(\mathfrak{D})$ be the unit sphere of $A(\mathfrak{D})$. By Strebel's frame mapping theorem, every Strebel point $[\mu]$ is represented by the unique Beltrami differential of the form $k|\varphi| / \varphi$, where $k=k_{0}([\mu]) \in(0,1)$ and $\varphi$ is a unit vector in $A_{1}(\mathfrak{D})$.

Two elements $\mu$ and $\nu$ in $L^{\infty}(\mathfrak{D})$ are infinitesimally equivalent, which is denoted by $\mu \approx \nu$, if $\iint_{\mathfrak{D}} \mu \phi d x d y=\iint_{\Delta} \nu \phi d x d y$ for all $\phi \in A(\Delta)$. Denote by $N(\mathfrak{D})$ the set 
of all the elements in $L^{\infty}(\mathfrak{D})$ which are infinitesimally equivalent to zero. Then $B(\mathfrak{D})$ $=L^{\infty}(\mathfrak{D}) / N(\mathfrak{D})$ is the tangent space of the space $T(\mathfrak{D})$ at the basepoint.

Given $\mu \in L^{\infty}(\mathfrak{D})$, we denote by $[\mu]_{B}$ the set of all elements $\nu \in L^{\infty}(\mathfrak{D})$ infinitesimally equivalent to $\mu$, and set

$$
\|\mu\|=\inf \left\{\|\nu\|_{\infty}: \nu \in[\mu]_{B}\right\} .
$$

We say that $\mu$ is infinitesimally extremal (in $[\mu]_{B}$ ) if $\|\mu\|_{\infty}=\|\mu\|$, uniquely infinitesimally extremal if $\|\nu\|_{\infty}>\|\mu\|$ for any other $\nu \in[\mu]_{B}$. We say that $\mu$ is infinitesimally non-decreasable if for any other $\nu \in[\mu]_{B}$, the set on which $|\nu(z)|$ $>|\mu(z)|$ has positive measure. Then $\mu$ is non-decreasable if it is uniquely extremal.

In a parallel manner we can define the boundary dilatation for the infinitesimal Teichmüller class $[\mu]_{B}$. The boundary dilatation $b\left([\mu]_{B}\right)$ is the infimum over all elements in the equivalence class $[\mu]_{B}$ of the quantity $b^{*}(\nu)$. Here $b^{*}(\nu)$ is the infimum over all compact subsets $F$ contained in $\mathfrak{D}$ of the essential supremum of the Beltrami coefficient $\nu$ as $z$ varies over $\mathfrak{D}-F$.

An infinitesimally equivalent class $[\mu]_{B}$ is called an infinitesimal Strebel point if $\|\mu\|>b\left([\mu]_{B}\right)$. It follows from the infinitesimal frame mapping theorem (see $[\mathbf{4}$, Theorem 2.4]) that if $[\mu]_{B}$ is an infinitesimal Strebel point, then there exists a unique vector $\varphi$ in $A_{1}(\mathfrak{D})$ such that $\mu$ and $\|\mu\||\varphi| / \varphi$ are infinitesimally equivalent.

\section{SOME PREPARATIONS}

For $\mu \in L^{\infty}(\Delta), \phi \in A(\Delta)$, let

$$
\lambda_{\mu}[\phi]=\operatorname{Re} \iint_{\Delta} \mu(z) \phi(z) d x d y .
$$

As is well known, a Beltrami coefficient $\mu$ is extremal if and only if it has a so-called Hamilton sequence, namely, a sequence $\left\{\phi_{n} \in A(\Delta):\left\|\phi_{n}\right\|=1, n \in \mathbb{N}\right\}$, such that

$$
\lim _{n \rightarrow \infty} \lambda_{\mu}\left[\phi_{n}\right]=\lim _{n \rightarrow \infty} \operatorname{Re} \iint_{\Delta} \mu \phi_{n}(z) d x d y=\|\mu\|_{\infty} .
$$

Given $\mu \in M(\Delta)$, let $f=f^{\mu}$ be the uniquely determined quasiconformal mapping of $\Delta$ onto itself with Beltrami coefficients $\mu$ and normalised to fix $1,-1$ and $i$.

Suppose that $\mu$ and $\nu$ are two equivalent Beltrami coefficients in $T(\Delta)$. Let $\tilde{\mu}$ and $\tilde{\nu}$ be the Beltrami coefficients of the quasiconformal mappings $f^{-1}$ and $g^{-1}$, respectively, where $f=f^{\mu}$ and $g=f^{\nu}$. Let $\mathfrak{J} \subset \Delta$ be a Jordan domain with $\overline{\mathfrak{J}} \subset \Delta$.

LEMMA 1. Let $\mu$ and $\nu$ be two equivalent Beltrami coefficients in $T(\Delta)$. In addition, suppose $\mu(z)=\nu(z)$ for almost every $z \in \Delta \backslash \overline{\mathfrak{J}}$. Then, $f^{\mu}(z)=f^{\nu}(z)$ for all $z$ in $\Delta \backslash \mathfrak{J}$ and hence $\widetilde{\mu}(w)=\widetilde{\nu}(w)$ for almost all $w$ in $f(\Delta \backslash \mathfrak{J})$. 
PROOF: For the sake of convenience, let $f=f^{\mu}$ and $g=f^{\nu}$. Let $\mu_{g \circ f^{-1}}(w)$ denote the Beltrami coefficient of $g \circ f^{-1}$. By a simple computation, we have

$$
\mu_{g \circ f^{-1}} \circ f(z)=\frac{1}{\tau} \frac{\mu(z)-\nu(z)}{1-\overline{\mu(z)} \nu(z)}
$$

where $\tau=\overline{f_{z}} / f_{z}$.

Thus, $\mu_{g \circ f^{-1}}(w)=0$ for almost all $w \in f(\Delta \mid \bar{J})$ and hence $\Psi=g \circ f^{-1}$ is conformal on $\Delta \backslash \overline{\mathfrak{J}}$. Since $\left.\Psi\right|_{S^{1}}=\left.g \circ f^{-1}\right|_{S^{1}}=i d$, we conclude that $\Psi=i d$ in $f(\Delta \mid \mathfrak{J})$. Thus, $\left.g\right|_{\Delta \backslash \bar{J}}$ $=\left.f\right|_{\Delta \backslash \bar{\jmath}}$. By the continuity of quasiconformal mappings, it follows that $\left.g\right|_{\Delta \mid J}=\left.f\right|_{\Delta \mid J}$. In addition, it is evident that $\tilde{\mu}(w)=\widetilde{\nu}(w)$ for almost all $w$ in $f(\Delta \backslash \mathfrak{J})$.

The following Reich's Construction Theorem is very useful. It was used by the author [10] to show that there exists $h$ such that all extremal quasiconformal mappings in $Q(h)$ are not of Teichmüller type.

Construction Theorem. ([6]) Let $A$ be a compact subset of $\Delta$ containing at least two points and such that $\Delta \backslash A$ is doubly connected. There exists a function $\alpha \in \mathrm{L}^{\infty}(\Delta)$ and a sequence $\varphi_{n} \in A(\Delta)(n=1,2, \ldots)$ satisfying the following conditions $(3.2)-(3.5)$ :

$$
\begin{gathered}
|\alpha(z)|= \begin{cases}0, & z \in A, \\
1, & \text { for almost all } z \in \Delta \backslash A,\end{cases} \\
\lim _{n \rightarrow \infty}\left\{\left\|\varphi_{n}\right\|-\lambda_{\alpha}\left[\varphi_{n}\right]\right\}=0, \\
\lim _{n \rightarrow \infty}\left|\varphi_{n}(z)\right|=\infty \quad \text { almost everywhere in } \Delta \backslash A .
\end{gathered}
$$

and as $n \rightarrow \infty$,

$$
\varphi_{n}(z) \rightarrow 0 \text { uniformly on } A \text {. }
$$

REMARK 2. Equation (3.5) is implied in the proof of Reich's Construction Theorem $[6]$.

From Reich's Construction Theorem, we can get

Lemma 2. Let $J \subset \Delta$ be a Jordan domain with $A=\bar{J} \subset \Delta$. Let $\alpha(z)$ and the sequence $\varphi_{n} \in A(\Delta)$ be constructed by Reich's Construction Theorem and let $\mu(z)$ $=k \alpha(z)$ where $k<1$ is a positive constant. Set

$$
\nu(z)= \begin{cases}\mu(z), & z \in \Delta \backslash A, \\ \beta(z), & z \in A,\end{cases}
$$

where $\beta(z)$ is in $M(J)$ with $\|\beta\|_{\infty} \leqslant k$. Then 
(1) $\nu(z)$ is extremal in $[\nu]$ and for any $\chi(z)$ extremal in $[\nu], \chi(z)=\nu(z)$ for almost all $z$ in $\Delta \backslash A$;

(2) $\quad \nu(z)$ is extremal in $[\nu]_{B}$ and for any $\chi(z)$ extremal in $[\nu]_{B}, \chi(z)=\nu(z)$ for almost all $z$ in $\Delta \backslash A$.

Proof: The proof of the first part of this lemma is the same as that of [10, Lemma 4] and the proof of the second part is included in that of [10, Theorem 3].

Recall that a Beltrami coefficient $\mu$ in $\mathfrak{D}$ is said to be locally extremal if for any domain $G \subset \mathfrak{D}$ it is extremal in its class in $T(G)$; in other words,

$$
\|\mu\|_{G}:=\underset{z \in G}{\operatorname{essup}}|\mu|=\sup \left\{\frac{\operatorname{Re} \iint_{G} \mu \phi(z) d x d y}{\iint_{G}|\phi(z)| d x d y}: \phi \in A(G)\right\} .
$$

Obviously, extremality in the whole domain is a prerequisite for a Beltrami coefficient to be locally extremal.

LEMMA 3. Using the notations of Lemma 2, then $\nu$ is locally extremal in $\Delta$ if and only if $\beta$ is locally extremal in $J$.

Proof: The necessary part is a fortiori. Now let $\beta$ is locally extremal in $J$. For given domain $G \subset \Delta$ with $G \backslash J \neq \emptyset$, by

$$
k \iint_{G \backslash J}\left|\varphi_{n}(z)\right| d x d y-\operatorname{Re} \iint_{G \backslash J} \mu(z) \varphi_{n}(z) d x d y \leqslant\left\|\varphi_{n}\right\|-\lambda_{\alpha}\left[\varphi_{n}\right],
$$

and Reich' Construction Theorem, we have

$$
\begin{aligned}
& \lim _{n \rightarrow \infty}\left(k \iint_{G}\left|\varphi_{n}(z)\right| d x d y-\operatorname{Re} \iint_{G} \nu(z) \varphi_{n}(z) d x d y\right) \\
& \leqslant \lim _{n \rightarrow \infty}\left(k \iint_{G \backslash J}\left|\varphi_{n}(z)\right| d x d y-\operatorname{Re} \iint_{G \backslash J} \mu(z) \varphi_{n}(z) d x d y\right) \\
& \quad+\lim _{n \rightarrow \infty}\left(k \iint_{J}\left|\varphi_{n}(z)\right| d x d y-\operatorname{Re} \iint_{J} \beta(z) \varphi_{n}(z) d x d y\right)=0 .
\end{aligned}
$$

Moreover, by equation (3.4) and Fatou's lemma,

$$
\lim _{n \rightarrow \infty} \iint_{G}\left|\varphi_{n}(z)\right| d x d y \geqslant \lim _{n \rightarrow \infty} \iint_{G \backslash J}\left|\varphi_{n}(z)\right| d x d y=\infty
$$

where the fact that $(G \backslash J)^{\circ} \neq \emptyset$ is needed. Thus,

$$
k-\frac{\operatorname{Re} \iint_{G} \nu(z) \varphi_{n}(z) d x d y}{\iint_{G}\left|\varphi_{n}(z)\right| d x d y} \rightarrow 0, n \rightarrow \infty,
$$

which indicates that $\nu(z)$ is extremal in its class in $T(G)$. Thus, $\nu$ is locally extremal in $\Delta$. 


\section{MAIN THEOREM}

By definition, the following lemma is evident.

LEMma 4. $\mu$ is an extremal-non-decreasable Beltrami coefficient in [ $\mu]$ if and only if for any other $\eta$ extremal in $[\mu]$, the set on which $|\eta(z)|>|\mu(z)|$ has positive measure.

Let $\Delta_{r}=\{z:|z|<r\}$ for $r \in(0,1)$. Choose $s=\frac{1}{4}, t=\frac{1}{2}$ and $A=\overline{\Delta_{t}}$.

LEMma 5. Let $\chi(z)$ be defined as follows,

$$
\chi(z)= \begin{cases}0, & z \in A-\Delta_{s}, \\ \tilde{k} & z \in \Delta_{s}\end{cases}
$$

where $\tilde{k}<1$ is a positive constant. Then $[\chi]$ as a point of the Teichmüller space $T\left(\Delta_{t}\right)$ of $\Delta_{t}$ contains infinitely many non-decreasable Beltrami coefficients $\eta$ with $\|\eta\|_{\infty}<\widetilde{k}$.

Proof: Let $s<r<t$. Note that $\chi(z)=0$ in $A \backslash \Delta_{s}$. When restricted to $\Delta_{r},[\chi]$ as a point of $T\left(\Delta_{r}\right)$ has the property $h([\chi])=0$ and hence is a Strebel point in $T\left(\Delta_{r}\right)$. Thus, by Strebel's frame mapping theorem, there exist $k_{r} \in(0,1)$ and a unit vector $\varphi_{r} \in A_{1}\left(\Delta_{r}\right)$ such that $k_{r}\left|\varphi_{r}\right| / \varphi_{r}$ and $\chi$ are equivalent in $T\left(\Delta_{r}\right)$. In addition, it is clear that $k_{r}<\tilde{k}$. Put

$$
\chi_{r}(z)= \begin{cases}0, & z \in A-\Delta_{r}, \\ k_{r} \frac{\left|\varphi_{r}(z)\right|}{\varphi_{r}(z)} & z \in \Delta_{r} .\end{cases}
$$

Then $\chi_{r}$ and $\chi$ are equivalent in $T\left(\Delta_{t}\right)$. Applying Lemma 1, it is easy to see that $\chi_{r_{1}}$ and $\chi_{r_{2}}$ restricted to $\Delta_{r_{2}}$ are equivalent in $T\left(\Delta_{r_{2}}\right)$ whenever $s<r_{1}<r_{2}<t$. Thus, $k_{r}$ is a strictly decreasing function as $r \in(s, t)$. Furthermore, we claim that $\chi_{r}$ is nondecreasable in $[\chi]$. Suppose to the contrary. Then there would exist $\eta$ in $[\chi]$ such that $|\eta(z)| \leqslant\left|\chi_{r}(z)\right|$ for almost all $z \in \Delta_{t}$. Obviously, $\eta(z)=\chi_{r}(z)=0$ on $A-\Delta_{r}$. Applying Lemma 1 again, we see that $\eta$ and $\chi_{r}$ restricted to $\Delta_{r}$ are equivalent in $T\left(\Delta_{r}\right)$. This happens if and only if $\eta=\chi_{r}$, which implies our claim. Thus, this lemma follows.

THEOREM 1. Let $A=\overline{\Delta_{t}}$ and let $\alpha(z)$ be constructed by Reich's Construction Theorem. Put $\mu(z)=k \alpha(z)$, where $k \in(0,1)$ is a constant. Set

$$
\nu(z)= \begin{cases}\mu(z), & z \in \Delta \backslash A \\ 0, & z \in A-\Delta_{s} \\ \tilde{k} & z \in \Delta_{s}\end{cases}
$$

where $\tilde{k} \in[0, k]$ is a constant. Then,

(1) when $\widetilde{k}>0,[\nu]$ contains infinitely many extremal non-decreasable Beltrami coefficients; 
(2) if $\tilde{k}=0$, then $\nu$ is the Beltrami coefficient in $[\nu]$ that is both uniquely locally-extremal (obviously, non-uniquely extremal) and uniquely extremalnon-decreasable.

And hence, if we set $h=f^{\nu}$, then either $Q(h)$ contains infinitely many extremal quasiconformal mappings with non-decreasable dilatations (when $\tilde{k}>0$ ) or admits an extremal quasiconformal mapping (but not uniquely extremal) that is both uniquely locallyextremal and uniquely extremal-non-decreasable (when $\widetilde{k}=0$ ).

Proof: First, let $0<\tilde{k} \leqslant k$. By Lemma 2, for any $\eta$ extremal in $[\nu], \eta(z)=\nu(z)$ almost everywhere on $\Delta \backslash A$. Then by Lemma $1, \eta(z)$ and $\nu(z)$ restricted to $\Delta_{t}$ are equivalent in $T\left(\Delta_{t}\right)$. Therefore, by Lemma 4 , if $\eta$ restricted to $\Delta_{t}$ is non-decreasable in its equivalence class $[\chi]$ (defined in Lemma 5 ), then it is non-decreasable in $[\nu]$ in $T(\Delta)$.

For $s<r<t$, put

$$
\nu_{r}(z)= \begin{cases}\mu(z), & z \in \Delta \backslash A, \\ 0, & z \in A-\Delta_{r}, \\ k_{r} \frac{\left|\varphi_{r}(z)\right|}{\varphi_{r}(z)} & z \in \Delta_{r} .\end{cases}
$$

where $k_{r}$ and $\varphi_{r}$ are from Lemma 5 . Then $\nu_{r}$ is an extremal non-decreasable dilatation in $[\nu]$ by Lemma 5 . Thus, (1) of Theorem 1 is proved.

Now, let $\widetilde{k}=0$. It follows directly from Lemmas 2,4 that $\nu$ is the element in $[\nu]$ that is uniquely extremal-non-decreasable. Since $\beta \equiv 0$ on $A$, as an immediate consequence of Lemma $3, \nu$ is locally-extremal in $[\nu]$. On the other hand, the uniqueness of local extremal follows clearly from Lemma 2 and the definition of local extremality.

REMARK 3. The example of local extremal (of course, instead of being uniquely extremal) given by Reich [5] has a constant modulus, whereas our example does not. The modulus of certain extremal Beltrami coefficients was discussed in a recent paper [12] of the author (joint with Yi Qi).

\section{INFINITESIMAL VERSION}

We have the infinitesimal version of Lemma 4 as follows.

LEMMA 6. $\mu$ is an infinitesimally extremal-non-decreasable Beltrami coefficient in $[\mu]_{B}$ if and only if for any other $\eta$ extremal in $[\mu]_{B}$, the set on which $|\eta(z)|>|\mu(z)|$ has positive measure.

LEMma 7. Let $\chi(z)$ be defined as in Lemma 5. Then $[\chi]_{B}$ as a point of the space $B\left(\Delta_{t}\right)$ of $\Delta_{t}$ contains infinitely many non-decreasable extremals $\eta$ with $\|\eta\|_{\infty}<\tilde{k}$.

The proof of Lemma 7 is a suitable modification from that of Lemma 5 except that the infinitesimal frame mapping criterion is used here. 
THEOREM 2. Let $\nu$ be the same as in Theorem 1. Then either $[\nu]_{B}$ contains infinitely many infinitesimally non-decreasable extremals when $0<\widetilde{k} \leqslant k$, or $\nu$ is the element in $[\nu]_{B}$ that is both uniquely locally-extremal (obviously, non-uniquely infinitesimally extremal) and uniquely infinitesimally extremal-non-decreasable if $\widetilde{k}=0$.

PROOF: By Lemmas 2, 6, 7, the proof almost takes word by word from that of Theorem 1 and so is skipped.

At last, we end this paper with an open problem.

PROBLEM 3. Does there exist $h$ such that each extremal quasiconformal mapping (of course, non-uniquely extremal) in $Q(h)$ has a non-decreasable dilatation?

If the answer is positive, then each extremal quasiconformal mapping in such $Q(h)$ is also locally extremal.

\section{REFERENCES}

[1] L.V. Ahlfors and L. Bers, 'Riemann's mapping theorem for variable metrics', Ann. Math. 72 (1960), 385-404.

[2] V. Božin, N. Lakic, V. Marković and M. Mateljević, 'Unique extremality', J. Anal. Math. 75 (1998), 299-338.

[3] N. Lakic, 'Strebel points', in Contemp. Math. 211 (Amer. Math. Soc., Providence, RI, 1997), pp. $417-431$.

[4] E. Reich, 'An extremum problem for analytic functions with area norm', Ann. Acad. Sci. Fenn. Ser. A. I. Math. 2 (1976), 429-445.

[5] E. Reich, 'On the uniqueness question for Hahn-Banach extensions from the space of $L^{1}$ analytic functions', Proc. Amer. Math. Soc. 88 (1983), 305-310.

[6] E. Reich, 'The unique extremality counterexample', J. Anal. Math. 75 (1998), 339-347.

[7] E. Reich, 'Extremal extensions from the circle to the disk', in Quasiconformal Mappings and Analysis, A Collection of Papers Honoring F. W. Gehring (Springer-Verlag, New York, Berlin, Heidelberg, 1997), pp. 321-335.

[8] Y. Shen and J. Chen, 'Quasiconformal mappings with non-decreasable dilatations', Chinese J. Contemp. Math. 23 (2002), 265-276.

[9] V.G. Sheretov, 'Locally extremal quasiconformal mappings', Soviet Math. Dokl. 21 (1980), 343-345.

[10] G.W. Yao, 'Is there always an extremal Teichmüller mapping?', J. Anal. Math. 94 (2004), 363-375.

[11] G.W. Yao, 'On extremality of two connected locally extremal Beltrami coefficients', Bull. Austral. Math. Soc. 71 (2005), 37-40.

[12] G.W. Yao and Y. Qi, 'On the modulus of extremal Beltrami coefficients', J. Math. Kyoto Univ. 46 (2006), 235-247.

Department of Mathematical Sciences

Tsinghua University

Beijing 100084

People's Republic of China

e-mail: gwyao@math.tsinghua.edu.cn 